Neurodegenerative Dis 2011;8:25-34

DOI: $\underline{10.1159 / 000313682}$
Received: December 23, 2009

Accepted after revision: April 14, 2010

Published online: August 4, 2010

\title{
Prenatal Flutamide Enhances Survival in a Myogenic Mouse Model of Spinal Bulbar Muscular Atrophy
}

\author{
Jamie A. Johansen ${ }^{\mathrm{a}}$ Sandra M. Troxell-Smith ${ }^{\mathrm{a}}$ Zhigang $\mathrm{Yu}^{\mathrm{e}}$ Kaiguo $\mathrm{Mo}^{\mathrm{c}}$ \\ D. Ashley Monks ${ }^{c, d}$ Andrew P. Lieberman ${ }^{\text {e S. Marc Breedlove }}{ }^{a, b}$ \\ Cynthia L. Jordan ${ }^{\mathrm{a}, \mathrm{b}}$ \\ ${ }^{a}$ Neuroscience Program and ${ }^{b}$ Department of Psychology, Michigan State University, East Lansing, Mich., USA; \\ 'Department of Psychology, University of Toronto at Mississauga, Mississauga, Ont., and ${ }^{\mathrm{d}}$ Institute for Medical

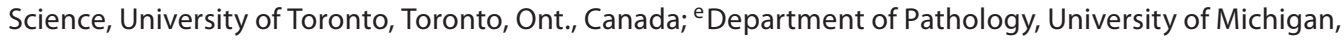 \\ Ann Arbor, Mich., USA
}

\section{Key Words}

Polyglutamine $\cdot$ Neuromuscular disease $\cdot$ Androgen .

Transgenic mice

\begin{abstract}
Background: Spinal bulbar muscular atrophy (SBMA) is caused by a CAG repeat expansion mutation in the androgen receptor (AR) gene, and mutant AR is presumed to act in motoneurons to cause SBMA. However, we found that mice overexpressing wild-type (wt) AR solely in skeletal muscle fibers display the same androgen-dependent disease phenotype as when mutant AR is broadly expressed, challenging the assumptions that only an expanded AR can induce disease and that SBMA is strictly neurogenic. We have previously reported that AR toxicity was ligand dependent in our model, and that very few transgenic (tg) males survived beyond birth. Methods: We tested whether the AR antagonist flutamide could block perinatal toxicity. tg males were treated prenatally with flutamide and assessed for survival and motor behavior in adulthood. Results: Prenatal treatment with flutamide rescued tg male pups from perinatal death, and, as adults, such perinatally rescued tg males showed an SBMA phenotype that was comparable to that of previously described untreated tg males. Moreover, tg males carrying a
\end{abstract}

mutant endogenous allele for AR - the testicular feminization mutation (tfm) - and thus having functional AR only in muscle fibers nevertheless displayed the same androgendependent disease phenotype as adults. Conclusions: These mice represent an excellent model to study the myogenic contribution to SBMA as they display many of the core features of disease as other mouse models. These data demonstrate that AR acting exclusively in muscle fibers is sufficient to induce SBMA symptoms and that flutamide is protective perinatally.

Copyright $\odot 2010$ S. Karger AG, Basel

\section{Introduction}

Spinal bulbar muscular atrophy (SBMA) is an X-linked disease characterized by progressive, slow loss of muscle strength that affects men, typically in midlife. The known cause of SBMA is an expansion of a trinucleotide CAG repeat that encodes a polyglutamine (polyQ) tract in the amino terminus of the androgen receptor (AR). Individuals with SBMA also typically exhibit signs of androgen insensitivity, such as gynecomastia, testicular atrophy and reduced fertility [1-5]. Similar CAG/polyQ expansion mutations in other genes have been linked to other

\section{KARGER}

(C) 2010 S. Karger AG, Basel

Fax +41 613061234 E-Mail karger@karger.ch www.karger.com www.karger.com/ndd
Jamie A. Johansen, $\mathrm{PhD}$

109 Zina Pitcher Place

4130 BSRB

Ann Arbor, MI 48109 (USA)

Tel. +1 734763 0616, Fax +1 734647 9777, E-Mail johanse8@msu.edu 
neurodegenerative diseases, including Huntington's disease, dentatorubral-pallidoluysian atrophy, and the spinocerebellar ataxias types $1,2,3,6,7,17$, but these other diseases are not male biased [6]. Unlike the affected proteins in these other polyQ diseases, the normal function of AR is relatively well understood, including its role in sexual differentiation and male reproduction $[7,8]$. For most polyQ diseases, a toxic gain of function through protein misfolding is thought to underlie disease, although a loss of normal function may also play a role in some cases [6]. The fact that both humans and rodents with a mutation in their $A R$ gene - the testicular feminization mutation $(\mathrm{tfm})$ - are androgen insensitive but are not prone to developing SBMA $[8,9]$ suggests that a toxic gain of AR function underlies the loss of motor function in SBMA.

It is widely assumed that mutant AR acts directly in motoneurons to trigger SBMA, and that muscle atrophy is a secondary response to disease originating in the motoneurons [10]. Our myogenic model of SBMA directly contradicts this scenario. In our mouse model, expression of a cDNA encoding a rat wild-type (wt) AR with only 22 glutamines is driven by the human skeletal $\alpha$ actin (HSA) promoter, resulting in high expression of AR only in skeletal muscle fibers. Unexpectedly, such transgenic (tg) mice show the same androgen-dependent loss of motor function [11] as other mouse models of SBMA [12-16]. Castration largely restores motor function of adult tg males, whereas testosterone $(\mathrm{T})$ treatment of otherwise asymptomatic adult tg females rapidly induces SBMA-like symptoms $[11,17]$. That overexpression of the wt form of a disease protein and the mutant form can induce comparable disease is not without precedence. Examples include tau implicated in Alzheimer's disease [18], $\alpha$-synuclein implicated in Parkinson's disease [19, 20], and ataxin in cerebellar ataxia type $1[21,22]$. What has yet to be shown is whether the mutant SBMA allele of AR acting only in motoneurons can induce an androgen-dependent loss of motor function. Mouse models expressing a neuron-specific expanded AR [23, 24] fail to develop a neurodegenerative phenotype, leading us to hypothesize that AR acts in muscles to cause SBMA.

Because the defining feature of SBMA in mouse models is an androgen-dependent loss of motor function [10], blocking androgen action would be expected to ameliorate disease symptoms. While there is a good deal of experimental data supporting this expectation, drugs such as flutamide that directly bind to AR to block its activation have thus far proven to have little therapeutic value for SBMA $[10,25]$. However, given the proven efficacy of flutamide in treating other androgen-dependent diseases such as prostate cancer [26], we decided to test flutamide in our model. We asked whether prenatal flutamide would enhance the perinatal survival of $\mathrm{tg}$ males. We now report that flutamide rescues tg males from perinatal death, and such 'rescued' males express the expected disease phenotype as adults. Furthermore, flutamide has these effects even in tg males carrying a dysfunctional endogenous allele for $A R(t \mathrm{fm})$ in which the only functional AR available to respond to the drug is in muscle fibers. These results demonstrate that AR activation in muscle fibers alone is sufficient for both perinatal death and symptoms of SBMA in our model.

\section{Materials and Methods}

Generation and Identification of $t g$ Mice

tg mice were generated and genotyped as previously described [11]. Animals were group housed, with water and food provided ad libitum. All animal procedures were approved and performed in compliance with the Michigan State University Institutional Animal Care and Use Committee, in accordance with the standards in the NIH Guide for the Care and Use of Laboratory animals.

Prenatal Flutamide Rescue of $t g$ Males

Timed pregnant dams from tg line 141 (L141) were injected subcutaneously with $5 \mathrm{mg}$ flutamide $/ 0.1 \mathrm{ml}$ propylene glycol at the nape of the neck on gestational days 15-20. Females were paired with males $\leq 18 \mathrm{~h}$ and the day females were mated was designated as day 0. Anogenital distance, an androgen-dependent measure, of resulting pups was measured at weaning to confirm that the dose of flutamide was effective. Motor function based on hang time and stride length of $9 \mathrm{tg}$ and $10 \mathrm{wt}$ male littermates was assessed starting at 5 weeks of age and continued weekly until sacrifice on 64-197 days.

Generation of $\mathrm{tfm} / \mathrm{tg}$ Males

A tg HSA-AR L141 male produced in the colony (without prenatal flutamide treatment) had sperm harvested for in vitro fertilization (University of Michigan Transgenic Animal Model Core, Ann Arbor, Mich., USA). Female tfm carriers on a C57Bl/ J6 background were superovulated and eggs harvested. After fertilization, embryos were transferred into pseudopregnant recipient B6D2F1/J mice. Four founding females were produced that carried both the transgene and the $t f m$ allele. Female offspring carrying both genes were bred with $\mathrm{C} 57 \mathrm{Bl} / \mathrm{J} 6$ males and treated with flutamide as described above during late gestation except that treatment began 1 day earlier (gestational days 14-20). Male offspring were of four different genotypes: wt, tfm, tg or, of particular interest to us, mice that had both the $t f m$ allele of the endogenous $A R$ gene and the transgene expressing wt AR in skeletal muscles (tfm/tg). We found that such $\mathrm{tfm} / \mathrm{tg}$ males are asymptomatic as adults because they have low circulating levels of T like tfm males $[\mathrm{tfm}=3.15 \pm 1.1 \mathrm{nmol} / \mathrm{l}($ mean $\pm \mathrm{SEM}, \mathrm{n}=7) ; \mathrm{tfm} / \mathrm{tg}=$ $1.12 \pm 0.26 \mathrm{nmol} / \mathrm{l}, \mathrm{n}=9 ; \mathrm{wt}=27.35 \pm 11.6 \mathrm{nmol} / \mathrm{l}, \mathrm{n}=6]$ [ [27]. 
Thus, once $\mathrm{tfm} / \mathrm{tg}$ males reached 120-124 days of age and still did not show a disease phenotype, we treated them (along with agedmatched tfm male controls also exposed to flutamide prenatally) with $\mathrm{T}$ to determine whether such $\mathrm{tfm} / \mathrm{tg}$ mice would express an SBMA phenotype in response to androgens when the only ARs available for activation are in muscle fibers. Four tfm males and $5 \mathrm{tfm} / \mathrm{tg}$ males were each implanted with one T-filled Silastic capsule (1.57 $\mathrm{mm}$ i.d., $3.18 \mathrm{~mm}$ o.d., effective release length of $6 \mathrm{~mm}$ ) under isoflurane anesthesia. Such T implants result in low physiological levels of circulating $\mathrm{T}$ in males [28]. Body weights, grip strength, rotarod, open-field cage activity and stride length were collected immediately prior to surgery (day 0 ), and on $1,3,5,7$, and 9 days of $\mathrm{T}$ treatment. On day 9 , after motor function was assessed, T capsules were removed and recovery of motor function was monitored on days 10, 12 and 15 (i.e. on 1, 3 and 6 days after $\mathrm{T}$ treatment ended).

\section{Behavioral Methods}

Hang test, stride length, grip strength and rotarod were measured as previously described [11, 17]. Cage activity was assessed by measuring the total amount of distance moved (i.e. path length in the horizontal plane) and the number of rears (i.e. number of vertical movements) in a novel environment (a $16 \times 16$ inch Plexiglas chamber) during a 5-min period, using the Versamax activity monitor (AccuScan Instruments, Columbus, Ohio, USA). The chamber was cleaned with $70 \%$ ethanol between tests. Data were analyzed using the Versamax software.

\section{Tissue Harvesting and Processing}

Animals ( $\mathrm{n}=8$ per group) were deeply anesthetized with isoflurane. Extensor digitorum longus (EDL) and anterior tibialis muscles were collected, weighed, placed in OCT-filled cryomolds, and frozen in liquid nitrogen. EDL muscles were cryostat (Leica) sectioned at $10 \mu \mathrm{m}$, and stored at $-80^{\circ} \mathrm{C}$ until stained. RNA was isolated from anterior tibialis muscles. Spinal columns were placed in buffered formalin for at least 30 days before harvesting the 5th lumbar (L5) ventral root. L5 ventral roots were embedded in Epon resin (Araldite 502: Poly/Bed 812: DDSA) and sectioned at $1 \mu \mathrm{m}$.

\section{Morphometrical Analysis}

Muscles and ventral roots were quantified as previously described [11]. Briefly, every fiber was counted in one cross-section taken from the belly of the EDL that was stained with hematoxylin and eosin (HE). Fiber size was measured from this same crosssection using Stereo Investigator (MicroBrightfield) to ensure unbiased sampling of muscle fibers. Alternate sections of EDL were stained with nicotinamide adenine dinucleotide (NADH). A third series was stained for AR. Motor axons were counted in a single cross-section of L5 ventral root and their size measured using Stereo Investigator as previously described [11].

\section{Gene Expression Analysis}

Total RNA isolated from anterior tibialis muscles with Trizol (Invitrogen) served as a template for cDNA synthesis using the High Capacity cDNA Archive Kit from Applied Biosystems. Gene-specific primers and probes labeled with a fluorescent reporter dye and quencher were used (Applied Biosystems) for acetylcholine receptor $\alpha$-subunit (AChR), myogenin and myogenic differentiation factor 1 (MyoD). TaqMan assays were performed us- ing 5-ng aliquots of cDNA from 3 tg and 4 wt males. Replicate tubes were analyzed for the expression of $18 \mathrm{~s}$ ribosomal RNA (rRNA) using a VIC-labeled probe. Threshold cycle values were determined by an ABI Prism 7900HT Sequence Detection System, and relative expression levels were calculated using the standard-curve method of analysis. Values are expressed relative to the control group. Vascular endothelial growth factor isoform 164 and 188 (VEGF) mRNA was measured as previously described [11] on 5 tg and 4 wt males. Briefly, total RNA was isolated from limb muscles using Trizol and analyzed using gel electrophoresis and spectrophotometry. Samples were DNase I treated prior to reverse transcription using a dT_20 VN primer (Sigma, Oakville, Ont., Canada) with SuperScript II. Resultant cDNA was diluted 1:8 for future use. Each cDNA reaction had a control reaction without reverse transcriptase. qPCR reactions were then assembled using SYBR Green Jumpstart Taq ReadyMix (Sigma) and VEGF primers (atcttcaagccgtcctgtgt and aatgctttctccgctctgaa) or GAPDH primers (caaggctgtaggcaaagtc and gaccacctggtcctctgtgt). Samples were incubated at $95^{\circ} \mathrm{C}$ for $10 \mathrm{~min}$ prior to thermal cycling ( 40 cycles of: $95^{\circ} \mathrm{C}$ for $30 \mathrm{~s}, 57^{\circ} \mathrm{C}$ for $30 \mathrm{~s}$, and $72^{\circ} \mathrm{C}$ for 30 s) using the Mx4000 System (Stratagene, La Jolla, Calif., USA). Melting curves were determined for all PCR products. The ROXnormalized fluorescence measurements were analyzed using the LinRegPCR program to correct for efficiency of each reaction. The expression of VEGF was normalized to the level of GAPDH within each sample.

\section{Statistics}

Results were analyzed using Statview 5.0.1. To assess the effects of prenatal flutamide on the probability of survival of $\mathrm{tg}$ males, a $\chi^{2}$ test was run that compared the proportion of tg males alive at weaning that were exposed prenatally to flutamide versus those from undisturbed litters. $t$ tests were used to assess the effects of the transgene on behavioral, cellular and molecular measures from flutamide-rescued tg males and their wt brothers. Because sibling pairings were not always perfectly matched, unpaired $t$ tests were used. In the second experiment, a two-way ANOVA was used to assess the effect of $\mathrm{T}$ treatment on the motor function of $\mathrm{tfm} / \mathrm{tg}$ males versus $\mathrm{tfm}$-only controls, with genotype as a between-subject independent measure, and time as a withinsubject, repeated measure. Unpaired t tests comparing the treatment groups were also done at individual time points for post-hoc tests when there were significant effects of time, treatment and/or significant interactions of these two factors. For all analyses, $\mathrm{n}=$ the number of animals in a group and results were considered significant if $\mathrm{p} \leq 0.05$

\section{Results}

\section{Prenatal Flutamide Rescues tg Males from Perinatal Death, but Does Not Prevent Expression of Motor Deficits in Adulthood}

We previously reported that tg mice expressing wt AR only in muscle fibers show androgen-dependent muscle pathology and motor axon loss in the tg line expressing high levels of AR (line 141). However, the majority of such 


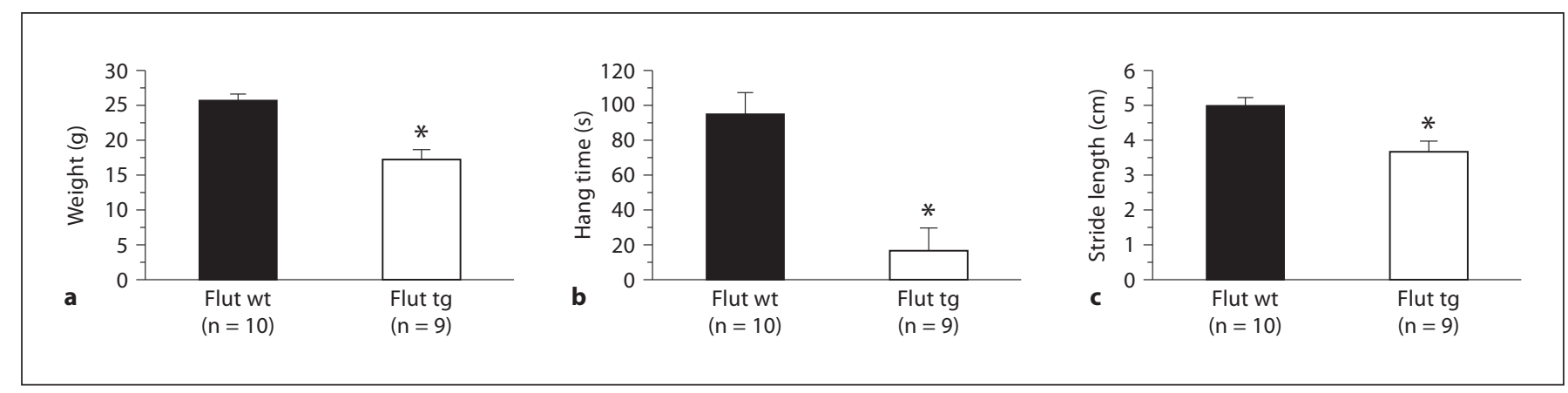

Fig. 1. tg males rescued by prenatal flutamide (Flut tg) display an SBMA phenotype as adults. Adult tg males exposed to prenatal flutamide weigh less (a), show reduced muscle strength (b) and a shortened stride (c) compared to similarly treated wt controls.
Mean age of wt males $=107$ days, range 65-197 days. Mean age of tg males $=111$ days (range 64-197 days). Plotted values are means \pm SEM. ${ }^{*} \mathrm{p}<0.05$.
Table 1. Number of L141 offspring alive at weaning based on 254 litters

\begin{tabular}{lll}
\hline & \multicolumn{2}{l}{ Offspring alive at weaning } \\
\cline { 2 - 3 } & $\begin{array}{l}\text { prenatal flutamide } \\
(\mathrm{n}=252)\end{array}$ & $\begin{array}{c}\text { no flutamide } \\
(\mathrm{n}=937)\end{array}$ \\
\hline wt male & $90(35.7 \%)$ & $311(33.2 \%)$ \\
tg male & $29^{\mathrm{a}}(11.5 \%)$ & $6^{\mathrm{b}}(0.6 \%)$ \\
wt female & $82(32.5 \%)$ & $340(36.2 \%)$ \\
tg female & $51(20.3 \%)$ & $280(30 \%)$ \\
\hline
\end{tabular}

a Significantly fewer $\operatorname{tg}$ males than predicted ( $\mathrm{p}<0.0001)$, but significantly more males than undisturbed litters $(\mathrm{p}<0.0001)$.

${ }^{\mathrm{b}}$ Significantly fewer tg males than predicted ( $\left.\mathrm{p}<0.0001\right)$.

tg males die perinatally in the high-expressing 141 line [11]. Thus, we treated pregnant dams carrying the AR transgene during late gestation with the antiandrogen flutamide to test whether flutamide could rescue tg males from this perinatal lethality. A $\chi^{2}$ test revealed that flutamide treatment rescued many tg male mice from death (table 1), significantly increasing the number of tg males surviving to weaning, compared to the proportion of tg males that survive when no prenatal flutamide is given $(\mathrm{p}<0.0001$, Cramer's V $=0.271)$. However, among the litters prenatally treated with flutamide, there were still fewer surviving tg males than would be expected by chance alone ( $\mathrm{p}<0.0001$, Cramer's $V=0.205)$. Prenatal flutamide did not alter plasma T levels in adulthood [flutamide-exposed wt and tg males $=21.0 \pm 5.7 \mathrm{nmol} / \mathrm{l}$ (mean \pm SEM of $\mathrm{n}=16$ ) vs. unexposed wt and tg males $=18.05 \pm 5.2 \mathrm{nmol} / \mathrm{l}($ mean \pm SEM of $\mathrm{n}=16)$, $\mathrm{p}=0.52]$. Moreover, flutamide-exposed tg and wt males exhibited the expected shortened anogenital distance at weaning, comparable to females (data not shown), confirming that this dose was effective in blocking activation of ARs necessary for prenatal masculinization of the external genitalia. This dose has been used to successfully feminize male rats [29-31]. Note that neither expression of the transgene nor flutamide affects survival of tg females (table 1).

Adult tg males prenatally rescued by flutamide show a reduced body weight (fig. 1a, $\mathrm{p}<0.0001$ ) compared to their wt brothers (which were also exposed to flutamide prenatally). Such flutamide-rescued tg males also show severely compromised motor function, like tg males from this same line (L141) that were not prenatally exposed to flutamide [11], being unable to hang for more than a few seconds on a wire grid (fig. $1 \mathrm{~b}, \mathrm{p}<0.0001$ ), and showing a significantly shortened stride (fig. $1 \mathrm{c}, \mathrm{p}<0.017$ ), comparable to the motor phenotype of the few untreated L141 tg males that survived to adulthood [11]. We also observed comparable deficits in motor function at all ages examined (as early as 5 weeks), indicating that even low prepubertal levels of $\mathrm{T}$ were sufficient to drive disease in our myogenic model.

Rescued tg males also show a significant decrease in both the number and cross-sectional area of muscle fibers in the EDL muscle (fig. $2 a, b, p<0.03$ ) compared to their wt brothers, in accord with previous data on untreated tg males. Rescued symptomatic tg males do not, however, show any significant losses in the number of L5 motor axons at the time of sacrifice (fig. $2 c, p=0.79$ ), but have significantly smaller motor axons in L5 (fig. $2 \mathrm{~d}, \mathrm{p}=$ 


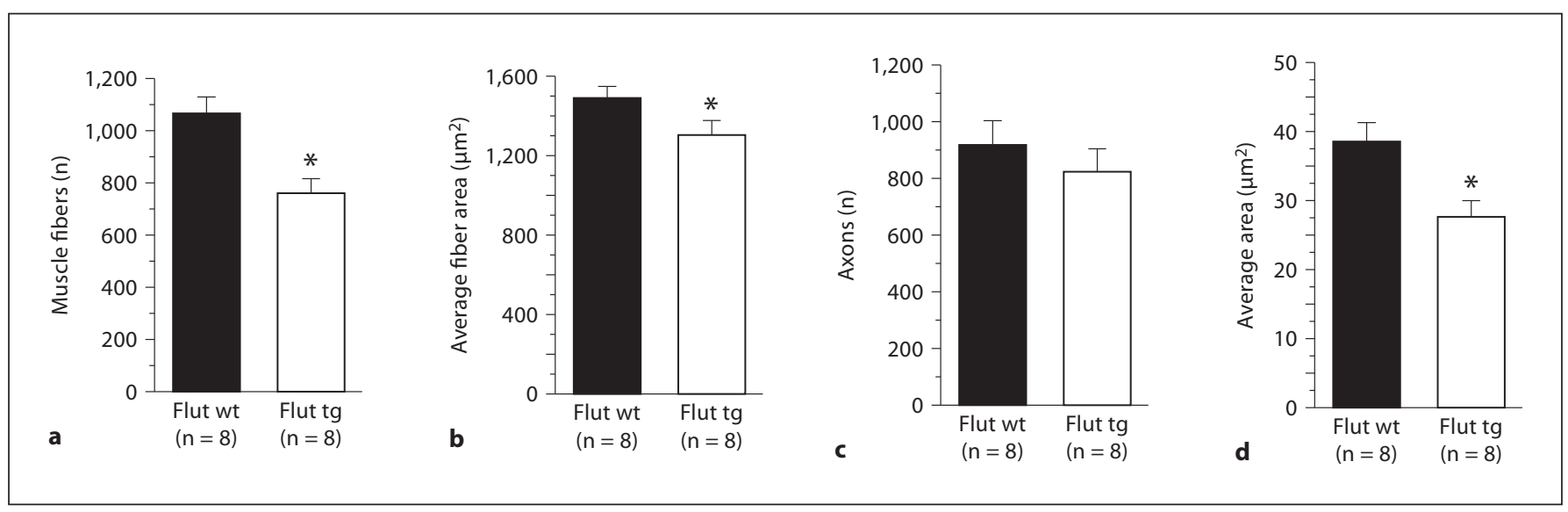

Fig. 2. Adult tg males rescued by prenatal flutamide (Flut tg) show regressive changes in skeletal muscle fibers and motor axons compared to wt control males. Adult tg males prenatally exposed to flutamide have significantly fewer (a) and smaller (b) muscle fibers in the EDL muscle compared to control-treated wt males (Flut wt). Although the number of L5 motor axons is not reduced in flutamide-rescued tg males compared to control treated wt males (c), cross-sectional area of L5 motor axons is decreased (d), suggesting their ensuing loss. Mean age of wt and tg brothers = 111 days, range 94-197 days. Plotted values are means \pm SEM. * $\mathrm{p}<0.05$.

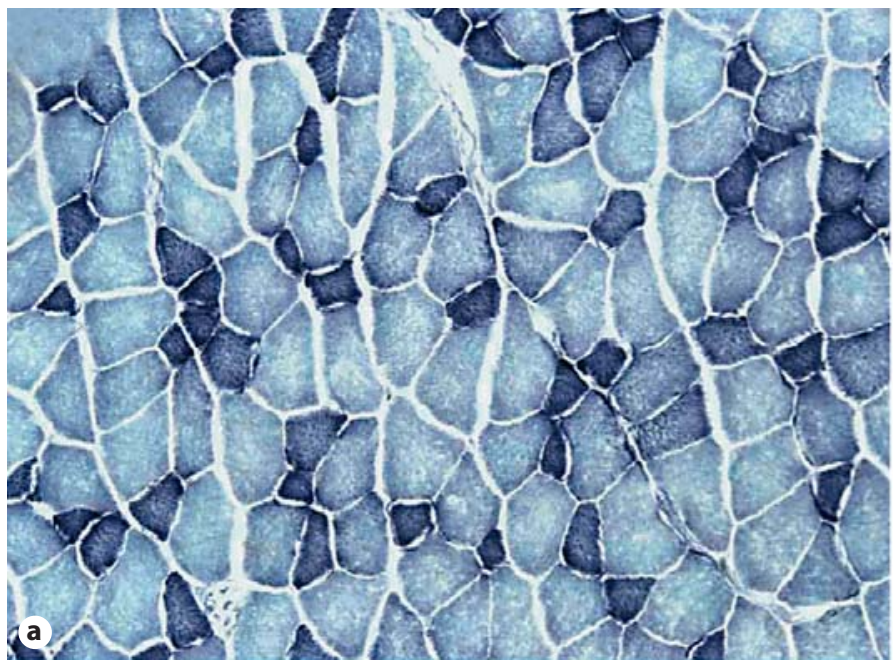

Fig. 3. NADH staining reveals expected pathology in adult EDL of flutamide-rescued tg males. Muscles from wt males also exposed prenatally to flutamide show the typical checkerboard pattern of staining (a), whereas muscles from flutamide-rescued

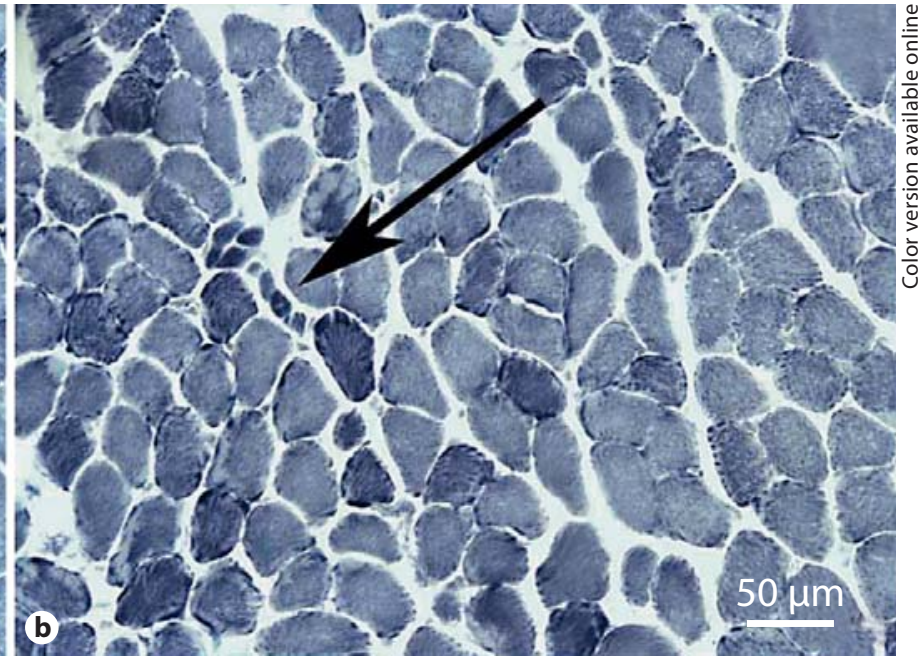

adult tg males show a generalized increase in staining intensity, suggesting an overall shift toward oxidative metabolism of diseased muscles (b). Such diseased muscles also contain small atrophic fibers (arrow).
0.0025), suggesting that motor axons may atrophy before their eventual loss. These data are in contrast to untreated tg males, which had significantly fewer motor axons but no apparent decreases in mean size [11].

EDL muscle fibers of tg males stained more darkly with NADH and showed a more uniform pattern of staining than the checkerboard pattern typical of wt EDL muscle (fig. 3), suggesting a generalized shift toward oxidative metabolism in tg muscles. We also observed occasional small atrophic fibers in the EDL from tg males (fig. 3). Such atrophic fibers were not evident in wt EDL. Such disease-related effects in muscle parallel those previously described for untreated tg [11]. 


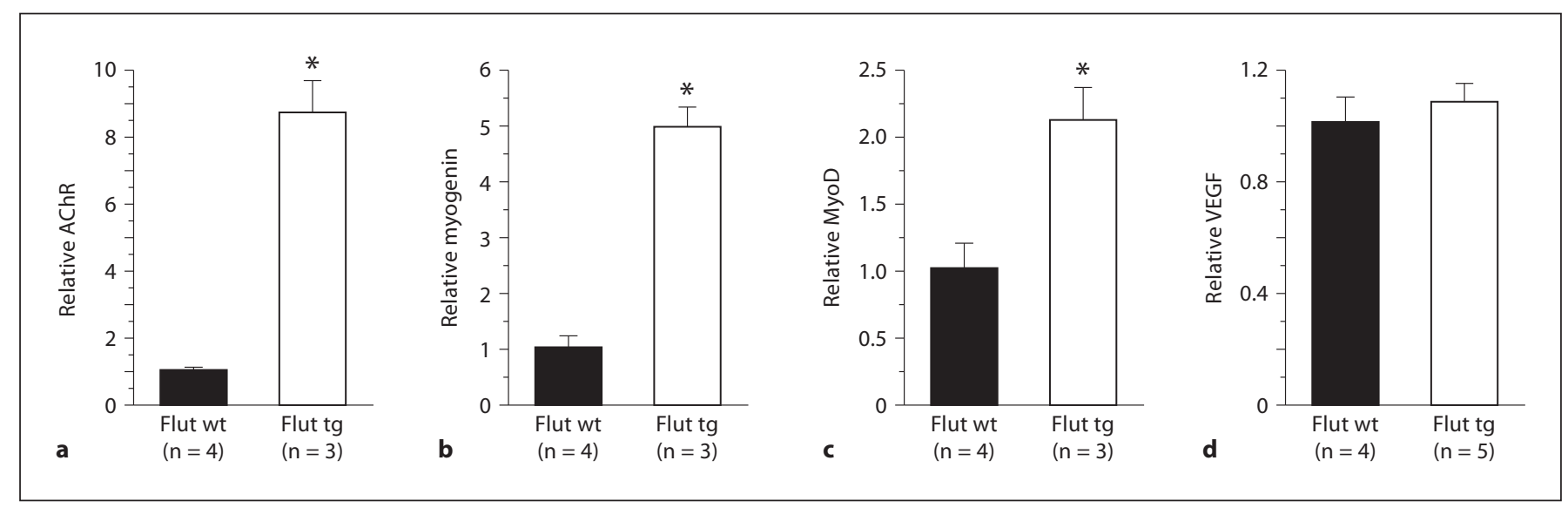

Fig. 4. Muscles from adult tg males rescued by prenatal flutamide (Flut tg) display denervation-like changes in gene expression. Levels of muscle mRNA for AChR $\alpha$-subunit (a) myogenin (b) and MyoD (c) are significantly upregulated in flutamide-rescued tg males (open bars) compared to their wt brothers also exposed to prenatal flutamide. In contrast, the level of muscle VEGF mRNA is not significantly different in rescued tg males compared to their wt brothers (d). Values are expressed relative to wt brothers. Age of wt and tg males = 114 days for AChR, MyoD and myogenin. For VEGF, mean age of tg males = 127 days (range 94-197 days) and mean age of wt males $=137$ days (range 114-197 days). Plotted values are means \pm SEM. ${ }^{*} \mathrm{p}<0.05$.
Quantitative RT-PCR revealed an upregulation in mRNA expression of AChR (fig. $4 \mathrm{a}, \mathrm{p}=0.0002$ ), myogenin (fig. $4 \mathrm{~b}, \mathrm{p}=0.0002$ ) and MyoD, fig. $4 \mathrm{c}, \mathrm{p}=0.0186$ ) in tg muscles compared to wt muscles, similar to the previously reported effects of the AR transgene on muscle gene expression in untreated tg males [11]. Importantly, because tg males in this study were compared to their wt male littermates which were also exposed to flutamide prenatally, these differences in gene expression between tg and wt male mice cannot be attributed to the flutamide treatment. However, we did not find the expected reduction in VEGF mRNA in muscle of flutamide-rescued tg males (fig. $4 \mathrm{~d}, \mathrm{p}=0.5$ ) as found previously for untreated tg males [11].

\section{tfm Male Mice Expressing tg AR in Their Skeletal}

Muscle Fibers (tfm/tg) Show an Androgen-Dependent

Loss of Motor Function

L141 tg males crossed onto a tfm background (tfm/tg males) lack functional AR throughout the body, including the brain and spinal cord, with one exception. They have functional AR exclusively in their skeletal muscle fibers due to expression of the AR transgene [11]. Prenatal flutamide treatment increased the number of $\mathrm{tfm} / \mathrm{tg}$ males that survived to weaning: a total of $7 \mathrm{tfm} / \mathrm{tg}$ males survived out of 8 litters (47 total pups) prenatally exposed to flutamide, while $0 \mathrm{tfm} / \mathrm{tg}$ males survived out of 31 litters (145 total pups) when no prenatal flutamide was given. $\mathrm{tfm} / \mathrm{tg}$ males, like $\mathrm{tfm}$ males, have an external pheno- type similar to females, with nipples and a short anogenital distance. Unlike females, but like $\mathrm{tfm}$ males, $\mathrm{tfm} / \mathrm{tg}$ males have undescended testes that are highly atrophic. Because adult $\mathrm{tfm}$ male mice have very low levels of $\mathrm{T}$ [27], $\mathrm{tfm}$ and $\mathrm{tfm} / \mathrm{tg}$ males were treated with $\mathrm{T}$ in adulthood to raise circulating levels to that of normal adult males.

Untreated adult $\mathrm{tfm} / \mathrm{tg}$ male mice had a slightly lower body weight than age-matched tfm males but exhibited normal motor function (on day 0 , fig. $5 \mathrm{a}-\mathrm{f}$ ). $\mathrm{tfm} / \mathrm{tg}$ males given $\mathrm{T}$ develop a profound neuromuscular phenotype, despite the fact that such males lack functional AR in their motoneurons. Androgen treatment induced a significant drop in both body weight and motor function. Interestingly, the effect on motor function was apparent before the effect on body weight. After only 1 day of $\mathrm{T}$ treatment, $\mathrm{tfm} / \mathrm{tg}$ males showed a significant reduction in general motor activity, as indicated by a significant decline in total distance moved in the open field (fig. 5c, $\mathrm{p}<0.04$ ), whereas significant losses in body weight were apparent only after 5 days of treatment (fig. 5 a). This decline in open-field activity was then followed by significant drops in the number of rears (fig. $5 b, p<0.0001$ ), grip strength (fig. $5 \mathrm{~d}, \mathrm{e}, \mathrm{p}<0.0001$ ) and stride length (fig. $5 \mathrm{f}$, $\mathrm{p}<0.01$ ), all evident by day 3 of treatment and all suggesting striking losses in muscle strength. This phenotype is similar to that described previously for untreated L141 tg males and T-treated tg females on a wt background [11, 17]. Furthermore, when $T$ treatment ceased on day $9, \mathrm{tfm} /$ tg males rapidly regained motor function and body weight 


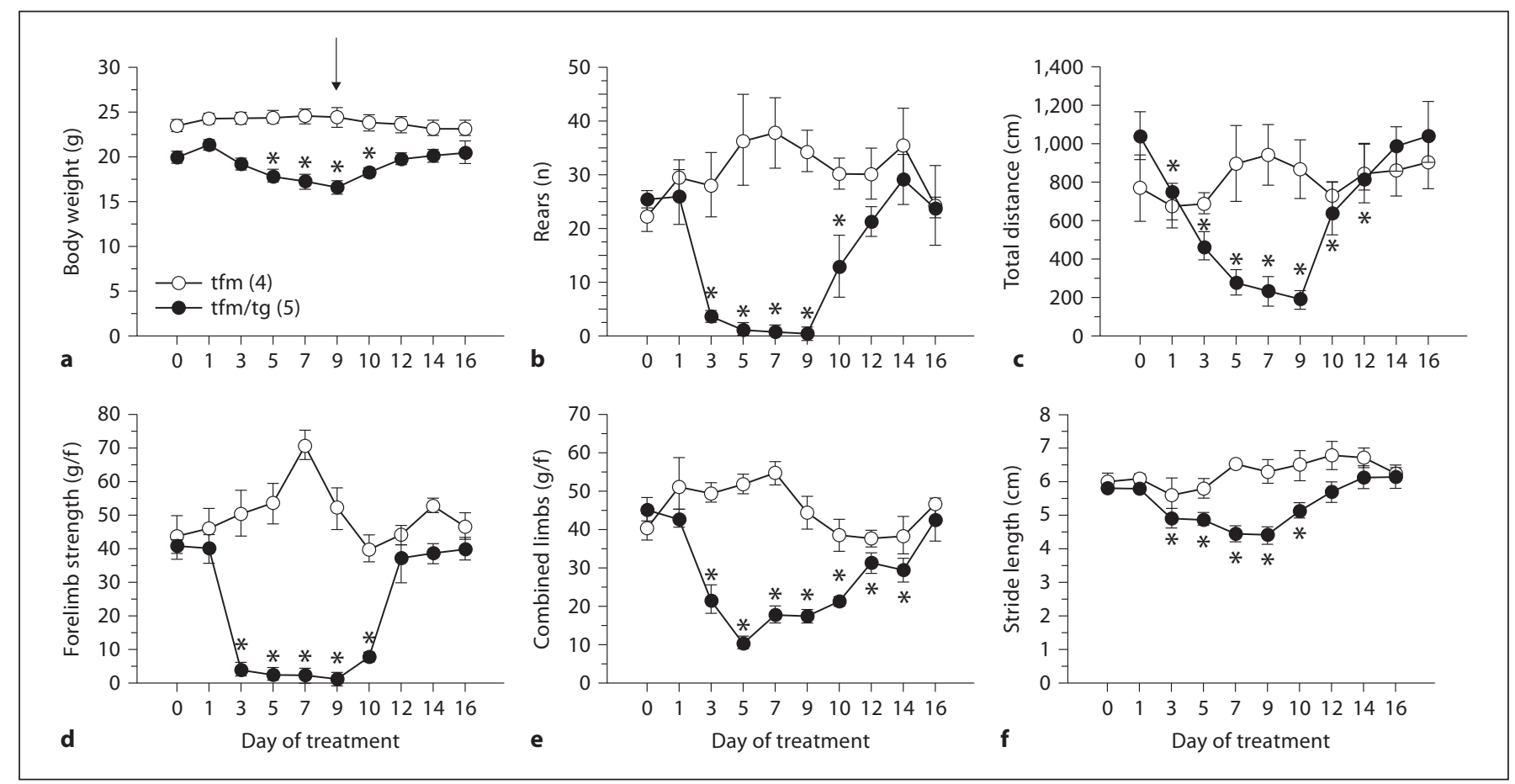

Fig. 5. Adult tfm males expressing functional AR only in muscle fibers (tfm/tg) show androgen-dependent loss of motor function. $\mathrm{tfm} / \mathrm{tg}$ males but not $\mathrm{tfm}$-only males ( $\mathrm{tfm}$ ) show the expected decline in body weight in response to $\mathrm{T}$ treatment begun on day 0 (a). Note that body weight quickly recovers in $\mathrm{tfm} / \mathrm{tg}$ males once $\mathrm{T}$ treatment ends on day 9 (arrow). Measures of motor function- based open field (b, c), grip strength (d, e) and stride length (f) also indicate that only $\mathrm{tfm} / \mathrm{tg}$ males and not $\mathrm{tfm}$ males show androgen-dependent losses and recovery of motor function. ${ }^{*} \mathrm{p}<$ 0.05 , significantly decreased from day 0 . Age of $\mathrm{tfm}$ and $\mathrm{tfm} / \mathrm{tg}$ males $=124$ days.

strongly expressing wt AR only in skeletal muscle fibers. We previously found that many of our tg males die perinatally, but those that survive show an androgen-dependent loss of motor function as adults [11]. While tg females show normal perinatal survival and motor function, androgen treatment of adult tg females induces a comparable disease phenotype to that of tg males [17]. Moreover, the deleterious effects of androgens on motor function in both males and females is fully reversible [11, 17], suggesting that cellular dysfunction underlies the motor deficits in our tg mice. The current report extends these findings by showing that prenatal flutamide rescued tg males from perinatal death. Furthermore, such adult tg males and adult tg males which survived perinatally without prenatal flutamide exhibited comparable behavioral, cellular and molecular pathologies. Therefore, these prenatally rescued tg male mice constitute an excellent model to study the role of muscle AR in SBMA. We also report that prenatal flutamide rescues tg males that have dysfunctional ARs elsewhere (because they have the $t f m$ allele of the endogenous $A R$ gene) and that 
such $\mathrm{tfm} / \mathrm{tg}$ males show the same androgen-dependent loss of motor function as tg males on a wt background. Because $\mathrm{tfm} / \mathrm{tg}$ males have functional ARs only in muscle fibers but nonetheless show androgen-dependent motor dysfunction characteristic of SBMA, we propose that AR acts exclusively in muscle fibers to trigger SBMA.

Prenatal flutamide likely rescues tg males from death by binding to ARs and blocking the effects of endogenous $\mathrm{T}$ [32-34]. However, blocking androgen action prenatally does not prevent expression of the disease later in life because flutamide-rescued tg males exhibit the same motor deficits in adulthood as untreated tg males. Such flutamide-rescued mice have fewer and smaller muscle fibers, smaller-caliber motor axons, and shorter life spans than wt controls, consistent with an SBMA disease phenotype. While flutamide-rescued tg males and untreated tg males did not show comparable axon loss [11], it is possible that the flutamide-rescued males would have eventually shown this loss. That flutamide-rescued males have smaller-caliber axons is consistent with the idea that axons are undergoing atrophy, foretelling their eventual loss.

Another difference we find between rescued tg males and untreated tg males is in VEGF mRNA levels in diseased muscle. VEGF was unaffected by disease in flutamide-rescued tg males, whereas we previously found a marked reduction in VEGF mRNA [11]. Thus, VEGF is probably not the signal causing motor axons to shrink in flutamide-rescued tg males. It is possible that exposure to prenatal flutamide masked the disease-related difference in muscle VEGF that was detected previously in untreated diseased tg males. Whether the expression of other muscle-derived neurotrophic factors is affected in our model remains an open question. Nonetheless, because muscle VEGF appears unaffected by disease in flutamide-rescued tg males, deficits in VEGF may be a rather late response to disease. Results in affected females also suggest this might be the case [17].

The phenotype shown by our flutamide-rescued tg males closely resembles the phenotype of another SBMA mouse model (described by Katsuno et al. [13]). Affected tg mice in this other model exhibit comparable androgendependent motor dysfunction that is accompanied by similar muscle and axonal pathology without motoneuronal death [13]. A key difference between this and our tg model is that an expanded ( 97 polyQ) full-length AR is broadly expressed in many cells, including motoneurons, whereas in our model, wt AR is expressed exclusively in muscle fibers. Nonetheless, both models exhibit remarkably similar androgen-dependent phenotypes, raising questions about the role of motoneuronal AR in SBMA.
Muscles from symptomatic prenatally rescued tg males show an upregulation in mRNA expression for AChR, MyoD, and myogenin-like muscles from symptomatic untreated tg males and androgen-treated tg females [11, 17]. Given that the same genes are also upregulated in denervated muscle $[35,36]$, our results suggest that ARs in muscle fibers trigger muscle denervation which may contribute to the loss of motor function in our diseased mice. It is noteworthy that these denervationlike changes in muscle gene expression are triggered by disease originating in muscle fibers, since this is where the transgene is expressed. Thus, data from our model indicate that pathology suggestive of neurogenic atrophy can have a muscular origin, and implicate muscles as a therapeutic target for effectively treating symptoms of SBMA.

While results in our tg mice suggest that AR acting only in skeletal muscle fibers is sufficient to trigger SBMA, it remained possible that expression of the disease in our model required activation of both $\mathrm{tg} \mathrm{AR}$ in muscle fibers and endogenous AR in motoneurons, since tg mice have the endogenous wt allele of the $A R$ gene. The present results from $\mathrm{tfm} / \mathrm{tg}$ males argue against this possibility. When the AR transgene is expressed in tfm males, such males have functional AR only in muscle fibers. Nonetheless, such tfm/tg males show the same androgen-dependent disease phenotype as tg males on a wt background, indicating that AR acting exclusively in skeletal muscle fibers is sufficient to account for the motor impairments that emerge in our mice. Evidently, activation of ARs in motoneurons is inconsequential. Because $\mathrm{tfm} /$ $\operatorname{tg}$ mice recover motor function when $\mathrm{T}$ treatment ceases, interfering with AR activity specifically in muscle fibers may offer an avenue for treating SBMA.

Background wt AR has been reported to be protective in another SBMA mouse model [37]. tfm mice expressing an expanded (100 polyQ), full-length human AR develop more severe disease symptoms than wt mice expressing the same 100 polyQ AR transgene, suggesting that normal AR might serve to protect cells from the toxicity of an expanded AR [37]. In contrast, we find little or no ameliorative effects of endogenous wt AR in our tg model. In fact, the time course of disease induced by $\mathrm{T}$ in our adult $\mathrm{tfm} / \mathrm{tg}$ males is remarkably similar to that induced by $\mathrm{T}$ in our $\mathrm{tg}$ females [17]; in each case, expression of the disease depends on androgens, and severe motor dysfunction develops within a week. Perhaps key differences, such as length of the glutamine tract ( 22 in our model vs. 100 ), or where tg AR is expressed (muscle fibers vs. broad expression), are behind the difference in the apparent role 
of endogenous $\mathrm{AR}$ in these two different tg models of SBMA.

The beneficial effects of flutamide in our model are contrary to previous findings in another SBMA mouse model [25]. However, we used a much higher dose of flutamide, which has been shown to be effective in blocking the masculinizing effects of androgens in rodents [29-31] (5 vs. $1.8 \mathrm{mg}$ per injection in Katsuno et al. [25]), and we gave flutamide daily rather than on alternate days. Given that injected flutamide is fully metabolized within $8 \mathrm{~h}$ [38], daily injections may be required to effectively avert SBMA symptoms.

The protective effects of flutamide also contrast with findings in Drosophila, where expression of a full-length 52 polyQ AR in the retina causes androgen-dependent neurodegeneration. Surprisingly, flutamide enhances rather than ameliorating neurodegeneration in this model [39]. Because flutamide blocked AR-activated transcription (mediated via androgen-responsive elements) but promoted nuclear translocation, nuclear translocation rather than activation of AR may critically mediate pathogenesis in Drosophila [39]. However, results from our model suggest that nuclear translocation is not sufficient to trigger disease. We suspect that AR activation is also somehow involved. A recent report on another SBMA mouse model reaches the same conclusion [40]. Because flutamide promotes androgen-dependent demise in Drosophila but prevents it in our model, apparently flutamide-bound AR behaves differently in the two systems. One possibility is that the Drosophila eye con- tains a different complement of AR cofactors than mouse muscle, causing flutamide to promote neurodegeneration in Drosophila but not mice, a scenario suggested by the opposing actions of tamoxifen in different tissues, an estrogen receptor antagonist that depends on different cofactors for these opposing actions [41]. The conflicting results of flutamide in cell models of SBMA also leave open the question of whether flutamide might be an effective therapeutic $[42,43]$.

Because flutamide rescues male mice from prenatal death, it is possible that it may also rescue motor function in our model, although this remains to be tested in adult tg male mice. Our model suggests that androgen-dependent motor dysfunction associated with SBMA can be caused by events that originate entirely in muscle, independent of AR action in motoneurons or other tissues. Therefore, blocking AR action in muscles may overcome the loss of motor function associated with SBMA while offering the significant advantage of preserving the beneficial effects of androgens in other tissues.

\section{Acknowledgments}

We thank David Jensen, Cindy Knaff, and Diane Redenius for their technical support.

This work was supported by National Institute of Health grants NS045195 (CLJ), NS51257 (DAM) and NS055746 (APL). Additional operating support provided by the MSU Foundation (CLJ). Trainee support was provided by a predoctoral fellowship (NS54517) from NIH (JAJ).

\section{References}

1 Arbizu T, Santamaria J, Gomez JM, Quilez A, Serra JP: A family with adult spinal and bulbar muscular atrophy, X-linked inheritance and associated testicular failure. J Neurol Sci 1983;59:371-382.

$\checkmark 2$ Dejager S, Bry-Gauillard H, Bruckert E, Eymard B, Salachas F, LeGuern E, Tardieu S, Chadarevian R, Giral P, Turpin G: A Comprehensive endocrine description of Kennedy's disease revealing androgen insensitivity linked to CAG repeat length. J Clin Endocrinol Metab 2002;87:3893-3901.

$\checkmark 3$ Kennedy WR, Alter M, Sung JH: Progressive proximal spinal and bulbar muscular atrophy of late onset. A sex-linked recessive trait. Neurology 1968;18:671-680.
4 Nagashima T, Seko K, Hirose K, Mannen T, Yoshimura S, Arima R, Nagashima K, Morimatsu Y: Familial bulbo-spinal muscular atrophy associated with testicular atrophy and sensory neuropathy (Kennedy-Alter-Sung syndrome). Autopsy case report of two brothers. J Neurol Sci 1988;87:141-152.

$\checkmark 5$ Sobue G, Hashizume Y, Mukai E, Hirayama M, Mitsuma T, Takahashi A: X-linked recessive bulbospinal neuronopathy. A clinicopathological study. Brain 1989;112(pt 1):209-232.

-6 Orr HT, Zoghbi HY: Trinucleotide repeat disorders. Annu Rev Neurosci 2007;30:575-621.

7 Morris JA, Jordan CL, Breedlove SM: Sexual differentiation of the vertebrate nervous system. Nat Neurosci 2004;7:1034-1039.

$\checkmark 8$ Wilson CA, Davies DC: The control of sexual differentiation of the reproductive system and brain. Reproduction 2007;133:331-359.
-9 Ono S, Geller LN, Lai EV: TfM mutation and masculinization versus feminization of the mouse central nervous system. Cell 1974;3: 235-242.

10 Jordan CL, Lieberman AP: Spinal and bulbar muscular atrophy: a motoneuron or muscle disease? Curr Opin Pharmacol 2008;8:752758.

11 Monks DA, Johansen JA, Mo K, Rao P, Eagleson B, Yu Z, Lieberman AP, Breedlove SM, Jordan CL: Overexpression of wild-type androgen receptor in muscle recapitulates polyglutamine disease. Proc Natl Acad Sci 2007;104:18259-18264.

12 McManamny P, Chy HS, Finkelstein DI, Craythorn RG, Crack PJ, Kola I, Cheema SS, Horne MK, Wreford NG, O'Bryan MK, De Kretser DM, Morrison JR: A mouse model of spinal and bulbar muscular atrophy. Hum Mol Genet 2002;11:2103-2111. 
-13 Katsuno M, Adachi H, Kume A, Li M, Nakagomi Y, Niwa H, Sang C, Kobayashi Y, Doyu M, Sobue G: Testosterone reduction prevents phenotypic expression in a transgenic mouse model of spinal and bulbar muscular atrophy. Neuron 2002;35:843-854.

-14 Yu Z, Dadgar N, Albertelli M, Scheller A, Albin RL, Robins DM, Lieberman AP: Abnormalities of germ cell maturation and Sertoli cell cytoskeleton in androgen receptor 113 CAG knock-in mice reveal toxic effects of the mutant protein. Am J Pathol 2006;168:195204.

-15 Sopher BL, Thomas PS Jr, LaFevre-Bernt MA, Holm IE, Wilke SA, Ware CB, Jin LW, Libby RT, Ellerby LM, La Spada AR: Androgen receptor YAC transgenic mice recapitulate SBMA motor neuronopathy and implicate VEGF164 in the motor neuron degeneration. Neuron 2004;41:687-699.

$\checkmark 16$ Chevalier-Larsen ES, O’Brien CJ, Wang H, Jenkins SC, Holder L, Lieberman AP, Merry DE: Castration restores function and neurofilament alterations of aged symptomatic males in a transgenic mouse model of spinal and bulbar muscular atrophy. J Neurosci 2004;24:4778-4786.

- 17 Johansen JA, Yu Z, Mo K, Monks DA, Lieberman AP, Breedlove SM, Jordan CL: Recovery of function in a myogenic mouse model of spinal bulbar muscular atrophy. Neurobiol Dis 2009;34:113-120.

-18 Wittmann CW, Wszolek MF, Shulman JM, Salvaterra PM, Lewis J, Hutton M, Feany MB: Tauopathy in Drosophila: neurodegeneration without neurofibrillary tangles. Science 2001;293:711-714.

-19 Singleton AB, Farrer M, Johnson J, Singleton A, Hague S, Kachergus J, Hulihan M, Peuralinna T, Dutra A, Nussbaum R, Lincoln S, Crawley A, Hanson M, Maraganore D, Adler C, Cookson MR, Muenter M, Baptista M, Miller D, Blancato J, Hardy J, Gwinn-Hardy $\mathrm{K}: \alpha$-Synuclein locus triplication causes Parkinson's disease. Science 2003;302:841.

-20 Sommer B, Barbieri S, Hofele K, Wiederhold K, Probst A, Mistl C, Danner S, Kauffmann S, Spooren W, Tolnay M, Bilbe G, van der Putten H, Kafmann S, Caromi P, Ruegg MA: Mouse models of $\alpha$-synucleinopathy and Lewy pathology. Exp Gerontol 2000;35: 1389-1403.

-21 Fernandez-Funez P, Nino-Rosales ML, de Gouyon B, She WC, Luchak JM, Martinez P, Turiegano E, Benito J, Capovilla M, Skinner PJ, McCall A, Canal I, Orr HT, Zoghbi HY, Botas J: Identification of genes that modify ataxin-1-induced neurodegeneration. $\mathrm{Na}$ ture 2000;408:101-106.
22 Tsuda H, Jafar-Nejad H, Patel AJ, Sun Y, Chen HK, Rose MF, Venken KJ, Botas J, Orr HT, Bellen HJ, Zoghbi HY: The AXH domain of ataxin-1 mediates neurodegeneration through its interaction with Gfi-1/ Senseless proteins. Cell 2005;122:633-644.

23 Bingham PM, Scott MO, Wang S, McPhaul MJ, Wilson EM, Garbern JY, Merry DE, Fischbeck KH: Stability of an expanded trinucleotide repeat in the androgen receptor gene in transgenic mice. Nat Genet 1995;9: 191-196.

24 Merry D, McCampbell A, Taye A, Winston RL, Fischbeck KH: Toward a mouse model for spinal and bulbar muscular atrophy: effect of neuronal expression of androgen receptor in transgenic mice. Am J Hum Genet 1996;59:A271.

25 Katsuno M, Adachi H, Doyu M, Minamiyama M, Sang C, Kobayashi Y, Inukai A, Sobue $\mathrm{G}$ : Leuprorelin rescues polyglutamine-dependent phenotypes in a transgenic mouse model of spinal and bulbar muscular atrophy. Nat Med 2003;9:768-773.

26 Antonarakis ES, Blackford AL, GarrettMayer E, Eisenberger MA: Survival in men with nonmetastatic prostate cancer treated with hormone therapy: a quantitative systematic review. J Clin Oncol 2007;25:49985008.

27 Zuloaga DG, Puts DA, Jordan CL, Breedlove SM: The role of androgen receptors in the masculinization of brain and behavior: what we've learned from the testicular feminization mutation. Horm Behav 2008;53:613626.

-28 Zuloaga DG, Morris JA, Jordan CL, Breedlove SM: Mice with the testicular feminization mutation demonstrate a role for androgen receptors in the regulation of anxietyrelated behaviors and the hypothalamicpituitary-adrenal axis. Horm Behav 2008;54: 758-766

29 Gladue BA, Clemens LG: Androgenic influences on feminine sexual behavior in male and female rats: defeminization blocked by prenatal antiandrogen treatment. Endocrinology 1978;103:1702-1709.

30 Casto JM, Ward OB, Bartke A: Play, copulation, anatomy, and testosterone in gonadally intact male rats prenatally exposed to flutamide. Physiol Behav 2003;79:633-641.

-31 Breedlove SM, Arnold AP: Hormonal control of a developing neuromuscular system. I. Complete demasculinization of the male rat spinal nucleus of the bulbocavernosus using the anti-androgen flutamide. J Neurosci 1983;3:417-423
32 Motelica-Heino I, Castanier M, Corbier P, Edwards DA, Roffi J: Testosterone levels in plasma and testes of neonatal mice. J Steroid Biochem 1988;31:283-286.

$>33$ Weisz J, Ward IL: Plasma testosterone and progesterone titers of pregnant rats, their male and female fetuses, and neonatal offspring. Endocrinology 1980;106:306-316.

34 Ward IL, Weisz J: Maternal Stress Alters Plasma Testosterone in Fetal Males. Science 1980;207:328-329.

-35 Klocke R, Steinmeyer K, Jentsch TJ, Jockusch H: Role of innervation, excitability, and myogenic factors in the expression of the muscular chloride channel ClC-1. A study on normal and myotonic muscle. J Biol Chem 1994;269:27635-27639.

>36 Kostrominova TY, Dow DE, Dennis RG, Miller RA, Faulkner JA: Comparison of gene expression of 2-mo denervated, 2-mo stimulated-denervated, and control rat skeletal muscles. Physiol Genomics 2005;22:227243.

$\checkmark 37$ Thomas PS, Fraley GS, Damien V, Woodke LB, Zapata F, Sopher BL, Plymate SR, La Spada AR: Loss of endogenous androgen receptor protein accelerates motor neuron degeneration and accentuates androgen insensitivity in a mouse model of X-linked spinal and bulbar muscular atrophy. Hum Mol Genet 2006;15:2225-2238.

38 Knobil E, Neill JD (eds): Encyclopedia of Reproduction. San Diego, Academic Press 1998, pp 171-172, 187.

>39 Takeyama K, Ito S, Yamamoto A, Tanimoto H, Furutani T, Kanuka H, Miura M, Tabata T, Kato S: Androgen-dependent neurodegeneration by polyglutamine-expanded human androgen receptor in Drosophila. Neuron 2002;35:855-864.

40 Montie HL, Cho MS, Holder L, Liu Y, Tsvetkov AS, Finkbeiner S, Merry DE: Cytoplasmic retention of polyglutamine-expanded androgen receptor ameliorates disease via autophagy in a mouse model of spinal and bulbar muscular atrophy. Hum Mol Genet 2009; 18:1937-1950.

-41 Shang Y, Brown M: Molecular determinants for the tissue specificity of SERMs. Science 2002;295:2465-2468

42 Walcott JL, Merry DE: Ligand Promotes intranuclear inclusions in a novel cell model of spinal and bulbar muscular atrophy. J Biol Chem 2002;277:50855-50859.

43 Darrington RS, Butler R, Leigh PN, McPhaul MJ, Gallo JM: Ligand-dependent aggregation of polyglutamine-expanded androgen receptor in neuronal cells. Neuroreport 2002; 13:2117-2120. 